\title{
A. GIAVATTO \& R. MULlER (éd.), Julien l'Empereur, Contre les Galiléens, texte, introduction, traduction et notes
}

Paris, 2018 (Bibliothèque des textes philosophiques), 248 p., ISBN

978-2-7116-2759-2

Marie-Odile Boulnois

\section{(2) OpenEdition}

Journals

Édition électronique

URL : https://journals.openedition.org/philosant/1918

DOI : 10.4000/philosant. 1918

ISSN : 2648-2789

Éditeur

Éditions Vrin

\section{Édition imprimée}

Date de publication : 31 octobre 2019

Pagination : 205-208

ISBN : 978-2-7574-2534-3

ISSN : 1634-4561

Référence électronique

Marie-Odile Boulnois, «A. giavatto \& R. muller (éd.), Julien l'Empereur, Contre les Galiléens, texte, introduction, traduction et notes », Philosophie antique [En ligne], 19 | 2019, mis en ligne le 23 avril 2019, consulté le 02 décembre 2022. URL : http://journals.openedition.org/philosant/1918; DOI https://doi.org/10.4000/philosant.1918

\section{(c) $\underset{\mathrm{EY}}{(\mathrm{NQ})} \mathrm{\Theta O}$}

Creative Commons - Attribution - Pas d'Utilisation Commerciale - Pas de Modification 4.0 International - CC BY-NC-ND 4.0

https://creativecommons.org/licenses/by-nc-nd/4.0/ 
justification » (p. xxiv). C'est donc à propos que l'auteur donne ensuite une présentation condensée, mais très claire, de certains points majeurs de la doctrine stoïcienne : sont ainsi passées en revue la conception stoïcienne de la nature, la vie en harmonie avec la nature qui rejoint la vie libre à laquelle invite Épictète, la distinction entre les biens, les maux et les indifférents, que recoupe et radicalise tout à la fois la distinction posée par ce dernier entre ce qui dépend de nous et ce qui ne dépend pas de nous, l'assimilation du bien à la seule vertu, ou encore la notion de prohairesis, que Long traduit par will, centrale chez Épictète puisqu'elle correspond justement à ce qui dépend de nous.

Mais si la mise en pratique de l'enseignement moral d'Épictète suppose d'adhérer à toute la doctrine de ce dernier, y compris à tout ce qu'elle peut avoir d'étranger à nos conceptions modernes, une telle mise en pratique pourrait bien s'avérer difficile. Long soutient cependant qu'il n'est pas requis d'adopter le déterminisme, le providentialisme et la théologie d'Épictète pour se conformer à son enseignement. Mais il ajoute immédiatement, et à raison nous semble-t-il, qu'il peut être pertinent de leur donner un sens adapté à notre époque : « his reverential stance toward divinity fits our time and place completely if we interpret it as sensitivity to the blessings of the ecosystem 》 (p. xxxviii). L'aperçu de la doctrine stoïcienne que propose l'auteur permettra au lecteur d'avoir l'information minimale nécessaire pour lire Épictète avec profit. Chaque fois qu'il rencontrera un terme technique, marqué par un astérisque, il pourra également se reporter au glossaire situé à la fin de l'ouvrage, qui, tout en allant à l'essentiel, est toujours précis et d'une grande aide. La traduction que donne Long du Manuel et des passages extraits des Entretiens est particulièrement claire, conformément au but qu'il s'est fixé (p. xlv). Parmi plusieurs remarques, qui méritent d'être lues, sur son travail de traduction (p. xlv-xlviii), Long note que les difficultés qu'il a rencontrées ont été d'ordre stylistique et rhétorique plutôt que lexical. Traduire le Manuel, en effet, ne suppose pas seulement d'en rendre le sens : il faut également tenter de lui conserver sa force de conviction et son pouvoir d'exhortation, pour qu'il soit à même d'encourager le lecteur à mettre en pratique ce qu'il lit. Au terme de sa lecture, ce dernier pourra consulter la bibliographie, habilement nommée Further readings, qui est succincte et par là même aisément maniable. Et il pourra considérer How to be free comme un abrégé d'éthique stoïcienne, comme un point de départ donc, mais aussi comme un texte qui est à portée de la main et auquel il est utile de revenir pour le lire encore.

Rodolphe Le Penru Université de Lille, STL

Angelo Giavatto \& Robert Muller (éd.), Julien l'Empereur, Contre les Galiléens, texte, introduction, traduction et notes, Paris, 2018 (Bibliothèque des textes philosophiques), 248 p., ISBN 978-2-7116-2759-2.

Cet ouvrage de poche présente une traduction française en regard du texte grec $\mathrm{du}$ Contre les Galiléens de l'empereur Julien. Cette ouvre perdue en tradition directe est conservée à travers les citations qu'en donne Cyrille d'Alexandrie dans les dix livres de son Contre Julien qui réfutent le premier livre de l'empereur. Du deuxième livre, il ne subsiste que des fragments, soit tirés des neuf livres suivants de Cyrille transmis de manière indirecte et très lacunaire, en grec ou en syriaque, soit tirés d'autres auteurs, en particulier Théodore de Mopsueste. La parution de cet ouvrage fait suite à celle de la grande édition critique du Contre Julien (Kyrill von Alexandrien, Gegen Julian. Teil 1. Buch 1-5, Ch. Riedweg (éd.), GCS NF 20, Berlin Boston : De Gruyter, 2016 ; Teil 2. Buch 6-10 und Fragmente, W. Kinzig et Th. Brüggemann (éd.), GCS NF 21, 
Berlin Boston : De Gruyter, 2017) et de la traduction française des livres III-V (Cyrille d'Alexandrie, Contre Julien, tome 2, III-V, introd. et annotation M.-O. Boulnois, trad. M.-O. Boulnois, J. Bouffartigue, P. Castan, texte grec Ch. Riedweg, SC 582, Paris : Éditions du Cerf, 2016). Néanmoins, A. G et R. M. ont choisi de suivre l'édition italienne de 1990 d'Emanuela Masaracchia sans véritablement tirer profit des parutions récentes.

L'introduction (p. 9-44) comprend quelques informations biographiques sur Julien (repères chronologiques, formation, apostasie), suivies d'une présentation du Contre les Galiléens (origine et nature, méthode de Cyrille). Une note (p. 38-41) indique que le texte grec est celui de Masaracchia et que la typographie distingue en petits caractères les passages où Cyrille résume Julien, pour les différencier des citations explicites ; certains choix de traduction sont justifiés (néanmoins, en dépit des explications données sur l'emploi d'une minuscule pour « dieu » lorsque Julien parle ou de la majuscule lorsqu'il s'agit de Cyrille, la règle n'est pas appliquée partout, voir p. 179). L'introduction se clôt par un plan fort utile donnant la structure du Contre les Galiléens. Il aurait pu mettre en évidence l'articulation en deux parties, annoncée (fr. 3) et répétée (fr. 58), qui accuse les Galiléens d'avoir d'abord trahi les Grecs pour suivre les Juifs, avant de leur reprocher de n'être même pas fidèles à ces derniers.

Pour compléter cette introduction, on aurait pu préciser que si « Julien connaît son sujet » (p. 33), ce qui est parfaitement exact, il est capable non seulement de citer abondamment la Bible, y compris des textes rarement invoqués ainsi que des variantes, mais aussi de faire référence aux controverses théologiques (voir fr. 62, 64, 80). La question des sources de la polémique est abordée (p. 35) avec la mention de Celse et de Porphyre. Mais rien n'est dit de l'hypothèse selon laquelle Julien aurait pu répondre au Contre Celse d'Origène dans plusieurs cas où Origène a précisément pris son adversaire en faute d'ignorance ou d'omission d'arguments.

Le texte grec et la traduction sont suivis par un appendice fournissant neuf autres extraits qui sont un ajout appréciable par rapport à l'édition de Masaracchia et utilisent les travaux de Guida, Bianchi et Trovato. Mais on ne voit pas quel critère a présidé au choix de ces textes, limité par rapport au tome 2 de la nouvelle édition des GCS, sinon la restriction aux textes grecs ou syriaques traduits en latin. Il est donc dommage de ne pouvoir disposer en traduction de la totalité de la documentation actuellement recensée, y compris des auteurs syriaques (traduits en allemand dans le volume 2 des GCS). L'annexe I présente un tableau comparant l'édition de Masaracchia à celle des GCS ; malheureusement il ne recense que les passages où le texte de Masaracchia n'a pas d'appui dans les manuscrits ou les éditions antérieures, sans en donner l'origine.

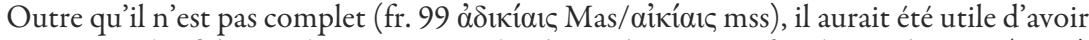
un récapitulatif de tous les écarts entre les deux éditions. De fait, l'introduction (p. 39) note que les différences sont peu significatives et n'ont pas d'incidence sur le sens, ce qui n'est pas tout à fait exact. Par exemple, p. 19 la note indique que des mots sont rétablis d'après la Septante, bien qu'ils soient absents du texte de Masaracchia. Or ces mots sont bien présents dans les manuscrits et n'ont pas besoin d'être « rétablis ». Au fr. 26

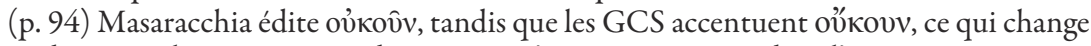
évidemment le sens puisque dans un cas c'est une question, dans l'autre une négation. Pour le fr. 33 (p. 104, 1. 16) la nouvelle édition contient un $\varphi \eta \sigma 1$, omis par Masaracchia, alors qu'il est très important, puisqu'il permet de confirmer qu'après un résumé, Cyrille redonne expressément la parole à Julien. Dans le fr. 69 Julien énumère différents types de sacrifices. Or Masaracchia, suivant Neumann, conjecture $\dot{x} \pi \dot{\varepsilon} \rho$ $\alpha \gamma v \varepsilon i ́ \alpha \varsigma$ qui est traduit « rites d'expiation », mais les manuscrits, suivis par la dernière édition, ont vं $\pi \dot{\varepsilon} \rho$ àyoías qui désigne un type de sacrifices offerts pour une faute commise dans l'ignorance 


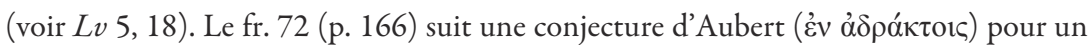
texte grec incompréhensible et la traduction donne « les juifs offrent des sacrifices chez eux ». La nouvelle édition choisit d'indiquer ce terme entre cruces. Une note aurait été nécessaire pour mettre en garde le lecteur sur l'incertitude du texte (il en est de même pour le fr. 26, p. 94 dont la fin pose des problèmes textuels qui ne sont pas mentionnés).

L'annexe II fournit un tableau commode mettant en regard les numérotations de Masaracchia, d'Aubert et de Migne. La bibliographie est succincte et surtout très générale. Elle aurait dû tenir compte des travaux récents publiés par les auteurs des nouvelles éditions sur tel ou tel fragment. Mentionnons quelques exemples. Le fr. 17 pose des problèmes de traduction et d'interprétation dont l'enjeu est de savoir si Julien reconnaît ou non aux récits bibliques la possibilité d'avoir un sens allégorique. Il faudrait renvoyer à Ch. Riedweg, « Mythos mit geheimem Sinn oder reine Blasphemie ? Julian über die mosaische Erzählung vom Sündenfall (Contra Galilaeos fr. 17, 10-12 Masaracchia) » dans A. Kolde, A. Lukinovich \& A-L. Rey (éd.), Kopvpaí å ỏvopí. Mélanges offerts à André Hurst, Genève, 2005, p. 367-375. Le fr. 21 aborde la question de la diversité des nations en lien avec le rôle des dieux ethnarques, sujet traité par J. Bouffartigue, « La diversité des nations et la nature de l'homme : l'empereur Julien et Cyrille d'Alexandrie dans une controverse incertaine », S. Crogiez-Pétrequin (éd.), Dieu(x) et hommes : histoire et iconographie des sociétés païennes et chrétiennes de l'Antiquité à nos jours. Mélanges en l'honneur de F. Thelamon, Rouen, 2005, p. 113-126. Dans les fr. 17, 30 et 33 Julien accuse le Dieu biblique de jalousie, ce qui met en question sa transcendance (M.-O. Boulnois, « Dieu peut-il être envieux ou jaloux ? Un débat sur les attributs divins entre l'empereur Julien et Cyrille d'Alexandrie », D. Auger et É. Wolff (éd.), Culture classique et christianisme. Mélanges offerts à Jean Bouffartigue, Picard, Paris, 2008, p. 13-25). Dans le fr. 70, Julien reproche aux chrétiens d'avoir abandonné les sacrifices juifs et donne l'exemple du bouc émissaire (Ch. Riedweg, « Exegese als Kampfmittel in der Auseinandersetzung zwischen Heiden und Christen. Zum "Sündenbock" von Leviticus 16 bei Julian und Kyrill von Alexandrien », Zeitschrift für Antikes Christentum 16, 2012, p. 439-476). Pour les auteurs anciens, les références sont données dans la Patrologie grecque, alors qu'il existe des éditions plus récentes. Pour Théodore de Mopsueste (fr. 92 à 95, 104 à 106), il faudrait renvoyer à A. Guida, Teodoro di Mopsuestia. Replica a Giuliano imperatore, Bologne, 1994 ; pour Jérôme (fr. 77 et 102), ses commentaires sont édités dans le Corpus Christianorum series latina (76 et 77).

La traduction est dans l'ensemble élégante et précise, et c'est sans doute sur ce point que cet ouvrage rendra le plus de services. Je formulerai seulement quelques remarques. Le fr. 18 rapporte une critique de Julien sur la cosmogonie mosaïque à laquelle il reproche d'être incomplète, car elle ne précise pas si les anges sont engendrés ou inengendrés. Il en va de même pour le $\pi v \varepsilon v \hat{\mu} \alpha$ de Dieu qui était porté au-dessus de l'eau $(G n 1,2)$. Les traducteurs ont choisi de rendre $\pi v \varepsilon v \hat{\mu} \alpha$ par « souffle ». Mais Julien qui connaît bien la théologie chrétienne sait parfaitement que ce verset est interprété commel'« Esprit »de Dieu, de sorte que cette traduction semble s'imposer. De manière étonnante, plusieurs formes à la première personne du singulier sont traduites à la troisième. Dans le fr. 19 है $\varphi \eta v$ est rendu par « selon son expression ». Ce passage pose le problème délicat des testimonia, c'est-à-dire des résumés de la pensée de Julien par Cyrille dans lesquels on peut se demander s'il reprend ou non la terminologie de l'empereur. Or dans ces lignes qui résument une thèse majeure de Julien selon laquelle le dieu des Hébreux ne possède pas le pouvoir sur l'univers, mais un empire restreint à la Judée, l'étude du vocabulaire montre qu'il s'agit plutôt d'une réécriture cyrillienne (c'est l'avis de Masaracchia contre Neumann, voir aussi SC 582, p. 14). En disant « comme je l'ai dit », Cyrille renvoie, 
non à Julien, mais à sa propre présentation de l'extrait de Julien sur la limitation du pouvoir du dieu des Hébreux (CJ III, 37, 652C). Il en va de même pour le fr. 21 (p. 81) où है $\varphi \eta v$ est traduit par « d'après ce qu'il a dit ». Dans ce passage, Cyrille rappelle que son adversaire suit les doctrines de Platon en y ajoutant les siennes propres. Cyrille dit donc « comme je l'ai dit » en renvoyant à ce qu'il a soutenu quelques lignes plus haut : « il fanfaronne, s'enorgueillit et ne s'attache qu'aux seules paroles de Platon » (CJ IV, 1). Le recours aux éditions récentes aurait permis d'éviter ces erreurs. Le fr. 67 commente l'épisode mystérieux de l'union des « fils de Dieu » avec des filles des hommes (Gn 6, 2-4) invoqué pour prouver que Moïse connaît une pluralité de fils de Dieu et non un Fils unique. Or pour Julien, ces « fils de Dieu » sont des anges. Au lieu de traduire : « celui qui parle de nombreux fils de Dieu et les appelle non pas hommes mais anges », il faut comprendre : « celui qui nomme une pluralité de fils de Dieu - et il ne s'agit pas

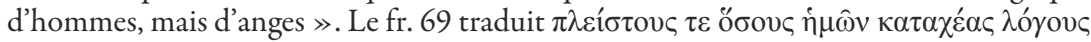
par « déverser en abondance des formules empruntées à notre doctrine », alors que le sens est « déverser contre nous la plus grande quantité possible d'arguments » (il s'agit d'une expression cyrillienne déjà utilisée en CJ I, 38 et VIII, 4).

En raison du rôle majeur de la Bible dans l'argumentation de Julien, on peut regretter l'absence de l'utilisation de G. Rinaldi, La Bibbia dei pagani, 2 vol., Bologne, 1998. De fait, comme le souligne Cyrille dans sa préface, le recours de l'empereur à la Bible donnait à son écrit une efficacité redoutable. Or l'index des lieux qui mêle toutes les références ne permet pas de se faire une idée précise de cette importance. De surcroît, certaines références bibliques manquent dans la traduction (fr. $14: G n 2,16$ et 3, 3 ; fr. $18: G n 1$, 2 ; fr. 19 : Dt 4, 19 ; fr. 26 : Gn 1, 3.6.9 ; fr. $35: S g 7,21$; fr. $74: G n 9,3$; fr. 97 : $M t 11,19$ et $L c 7,34$ ). Il faudrait aussi corriger quelques références (le fr. 65 ne cite pas $O s$ 13, 4 mais $I s 43,11$; le fr. 93 qui fait allusion au jeûne de Moise ne renvoie pas à $E x 24,18$ mais à $E x 34,28$ ). L'ajout de guillemets dans le fr. 73 rapportant l'épisode de Phinees aurait permis de montrer que Julien ne se contente pas de raconter l'histoire mais reprend textuellement des expressions bibliques, comme il le signale lui-même en disant $\varphi \eta \sigma \mathrm{l}$. À propos du texte biblique cité par Julien, une remarque de l'introduction est assez étonnante (p. 34) : «Les citations qui parsèment son ouvrage sont fidèles et conformes, à quelques détails près, au texte que nous lisons aujourd'hui dans la Bible grecque ». Mais de quelle «Bible grecque » parle-t-on ? Outre qu'il peut y avoir des citations de mémoire, la comparaison des leçons de Julien doit être menée avec toutes les variantes de la tradition manuscrite de la Septante. L'annotation n'est pas toujours

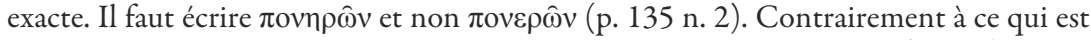
dit, le « symbole des Apôtres » n’a pas été défini par le concile de Nicée (p. 147).

En dépit de ces remarques, il faut saluer l'intérêt de cette publication qui fournit dans un format accessible, y compris financièrement, une traduction française rigoureuse et très lisible d'un ouvrage majeur, comme en témoigne sa postérité tant dans l'Antiquité tardive que jusqu'à l'époque moderne.

Marie-Odile Boulnois École Pratique des Hautes Études, Université PSL, LEM

Frédéric FAUQuIER, Le Parménide au miroir des platonismes. Logique-Ontologie-Théologie Paris, Les Belles Lettres, 2018 (Collection d'études anciennes. Série grecque, 157), 546 p., ISBN 978-2-251-44827-5.

Interroger la diversité des lectures antiques du Parménide pour dégager l'unité et la singularité du néoplatonisme, telle est l'ambition, pleinement tenue, du beau livre 